\title{
DER DOPPELTE ALEXANDER DER GROSSE?
}

\section{SABINE MÜLLER \\ CHRISTIAN-ALBRECHTS-UNIVERSITÄT ZU KIEL}

smueller@email.uni-kiel.de

Article received on 29.01.2011

Accepted on 19.05.2011

\begin{abstract}
ZUSAMMENFASSUNG
Basierend auf einer antiken Tradition über ihren gemeinsamen Besuch der gefangenen Familie des persischen Großkönigs wurde Hephaistion, der engste Gefährte und Vertraute Alexanders des Großen, in der Rezeption durch europäische Künstler der Renaissance und des Barock als sein äußerlich identischer Doppelgänger gezeigt. Antiker Hintergrund war eine Darstellung der beiden im Sinne der aristotelischen Freundschaftslehre, die von einer Seele in zwei Körpern ausgeht. In der Rezeption des 20. und 21. Jahrhunderts in Populärwissenschaft und Populärkultur erfährt die Darstellung des doppelten Alexanders weitere Facetten: die Abwertung Hephaistions als sein opportunistischer Schatten, die Betonung von Alexanders Hinwendung zur aristotelischen Philosophie und zum heroischen Ideal oder die Darstellung seiner inneren Zerrissenheit.
\end{abstract}

\section{SCHLAGWÖRTER}

Doppelgänger, Alexander III. von Makedonien, Hephaistion, Aristoteles, antike Freundschaftslehre, Curtius, europäische Kunst, Ludwig XIV., historischer Roman, Film.

\section{THE DOUBLE ALEXANDER THE GREAT?}

\section{ABSTRACT}

On the basis of an ancient tradition on their visit to the captured royal family of the Persian great king, Hephaistion, the closest friend and confidant of Alexander the Great, was depicted in European art of the Renaissance and Baroque as his look-alike. The ancient background was Aristotle's theories on friendship. During the 20th and 21st centuries the image of the doubled Alexander received further treatment. Hephaistion was shown as a mere shadow of Alexander and opportunist while Alexander was depicted as a true philosopher king and hero or as a person at odds with himself.

\section{KEYWORDS}

Double, Alexander III of Macedonia, Hephaistion, Aristotle, ancient theory of friendship, Curtius, European art, Louis XIV, historical romance, movie.

\section{EINLEITUNG}

In einer berühmten Episode aus dem Leben des Herrschers und Eroberers Alexander III. von Makedonien erscheint sein engster Gefährte, der makedonische Adlige Hephaistion aus Pella, als sein Alter Ego. Am Tage nach dem zweiten makedonischen Sieg 
über die persischen Heere, 333 v. Chr. bei Issos, sollen beide zusammen das königliche Zelt der in Gefangenschaft geratenen Familie Dareios' III., der sich auf dem strategischen Rückzug befand, betreten haben. Dabei kam es zu einer Verwechslung; die persische Königinmutter Sisygambis hielt Hephaistion für Alexander. Als der Irrtum aufgeklärt wurde, soll Alexander ohne Groll reagiert und stattdessen erklärt haben, auch Hephaistion sei Alexander.

Dieses Doppelgängerthema in der Alexandergeschichte, das von der aristotelischen Freundschaftslehre gefärbt war, entwickelte sich in der Alexanderrezeption, insbesondere in der europäischen Kunst der Renaissance und des Barock, zum beliebten Bildsujet und moralischen exemplum für Milde, Großmut und Mäßigung (Noll 2005: 32; Hadjinicolaou: 1997, 19). Dabei wurde Hephaistion, wie in den zu besprechenden Werken von Giovanni Antonio Bazzi, Charles LeBrun und Paolo Veronese, im Unterschied zur antiken Tradition, in der von einer ideellen Doppelgängervorstellung ausgegangen wird, als äußerlich mit Alexander identisch dargestellt.

Im Folgenden wird die Rezeption der Darstellung Hephaistions als Alexanders Alter Ego in ausgewählten Werken der historischen Biographie und der Populärkultur des 20. und 21. J ahrhunderts analysiert. Zur Untersuchung stehen die Romane Alexander. Roman einer Utopie von Klaus Mann (1929/30), La jeunesse d' Alexandre von Roger Peyrefitte (1977), die Alexanderbiographien von Fritz Schachermeyr (1973) und Robin Lane Fox (1974) sowie der Film Alexander the Great (2004) unter der Regie von Oliver Stone. Als Ausgangspunkt werden die Hintergründe des Doppelgängerthemas in den antiken Quellen zur Alexandergeschichte und die Entwicklung und Wandlung des Themas des doppelten Alexanders in ausgewählten Werken der europäischen Kunst der Renaissance und des Barock kurz aufgezeigt.

\section{HEPHAISTIONS LAUFBAHN}

Hephaistion aus Pella, Sohn des Amyntor, war ein makedonischer Adliger, über dessen Familie nichts weiter bekannt ist. Es wird angenommen, sie habe vor Hephaistions Aufstieg unter Alexander keinen größeren Einfluss gehabt (Reames-Zimmerman 1998: 67). Für die schon in der Antike viel zitierte Jugendfreundschaft von Alexander und Hephaistion, ihr gemeinsames Aufwachsen am Hof und den Unterricht durch Aristoteles in Mieza gibt es nur spätere, teilweise in ihrer Glaubwürdigkeit umstrittene Quellen. So wird ein Brief des Philosophen an Hephaistion in einem Schriftenverzeichnis erwähnt (Diog. Laert. 5,27), was jedoch nicht impliziert, dass er auch sein Schüler gewesen war. Es ist zwar möglich, dass Hephaistions Familie zu den factions an Philipps Hof gehörte, die Alexanders Herrschaftsansprüche unterstützten, da diese sich überwiegend aus Olympias' mit ihrer Heimat Epeiros verbundenen Klientel oder weniger bedeutenden Adelsfamilien aus Niedermakedonien zusammensetzten (Müller 2003: 31-2; Heckel 1985: 289). Fassbar wird eine enge Verbindung der beiden jedoch erst in der zweiten Phase von Alexanders Regierung, ab 330 v. Chr. Zu diesem Zeitpunkt begann Hephaistions Aufstieg und 
Sichtbarkeit: Nach unspektakulären militärischen Anfängen wurde ihm in einem gewaltigen Karriereschub die Leitung der Hälfte der Eliteeinheit der Hetairenreiterei zuteil (Diod. 18,3,4). Danach erhielt er immer öfter die Verantwortung über den Hauptteil des Heers (Arr. an. 6, 2,2. 21,3-4. 28,7; 7,7,1; Curt. 9,10,6). Nach der Rückkehr vom Indienfeldzug zählte er 324 v. Chr. zu denjenigen, die im Rahmen der Ehrungen für ihre Verdienste besonders hervorgehoben wurden (Arr. an. 7,5,6; Diod. 17,93,1).

$\mathrm{Zu}$ einem ungewissen Zeitpunkt wurde er ins prestigiöse Amt eines der sieben Somatophylakes, der herrscherlichen Leibwache, berufen (Arr. an. 6,28,3-4; vgl. Heckel 2009: 133). Ebenso unsicher ist, wann zwischen 330 und 324 v Chr. seine Ernennung zum Chiliarchen (Arr. Succ. 1a,3) erfolgte. Über die Kompetenzen, die Hephaistion in dieser Funktion innehatte, schweigen die Quellen. Aktuell wird im Gegensatz zur älteren Forschungsmeinung, es handle sich um einen mit der persischen Chiliarchie identischen Aufgabenbereich, ${ }^{1}$ angenommen, dass das Amt trotz des persischen Namens primär mit Hephaistions Oberbefehl über die makedonische Hetairenreiterei zu tun hatte (Arr. an. 7,14,10; vgl. Meuus 2009: 308-9). Flexibel gestaltet (Briant 2009: 74), sollte es vielleicht seine herausgehobene Vertrauensposition bei Alexander auch institutionell unterstreichen.

Hephaistions besondere Rolle am Hof wurde sinnfällig hervorgehoben, als er 324 in Susa auch noch Alexanders Schwager wurde. Sie heirateten parallel achaimenidische Schwestern (Arr. an. 7,4,5), was auch eine dynastische Anbindung Hephaistions an Alexander mit sich brachte (Wirth 1967: 1023). Sein steiler Aufstieg endete abrupt mit seinem frühen Tod im Spätsommer 324 in Ekbatana. Die Ursache war wohl eine fiebrige Erkrankung, kombiniert mit einer Alkoholvergiftung und körperlicher Erschöpfung (Arr. an. 7,14,1; Plut. Alex. 72,1-2. Diod. 17,110,7-8; J ust. 12,12,11-12; vgl. Wirth 1993: 324, Anm. $245,347)$.

\section{DER DOPPELTE ALEXANDER IN DER ANTIKEN ÜBERLIEFERUNG}

Drei Hauptquellen zur Alexandergeschichte berichten von der Verwechslung Alexanders mit Hephaistion im Zelt der Familie des persischen Königs: Quintus Curtius Rufus, Diodor und Arrian. ${ }^{2}$ Curtius, Verfasser der einzig bekannten antiken Alexandermonographie in lateinischer Sprache, Historiae Alexandri Magni Macedonis, schreibt:

... ohne die Schar seiner Begleiter einzulassen, trat er allein mit Hephaistion in das Zelt. Dieser war ihm unter allen seinen Freunden bei weitem der liebste, war mit ihm zusammen erzogen und Mitwisser aller seiner Geheimnisse. Auch hatte kein anderer das

1 Die Kompetenzen des persischen Chiliarchen sind ebenfalls umstritten. Laut griechischer Quellen hatte er ein militärisches Kommando inne (Hdt. 7,81; Xen. Kyr. 2,1,23), regelte zudem den Zugang zu Audienzen, kontrollierte die Leibwache und die Termine des Herrschers (Ael. VH 1,21; Nep. 9,3,3).

2 Vgl. Val. Max. 4,7,ext.2 A. 
Recht, ihn so freimütig zu ermahnen, dieses Recht übte er aber so aus, dass es ihm mehr vom König zugestanden, als von ihm selbst in Anspruch genommen zu sein schien. Und war er ebenso alt wie Alexander, übertraf er ihn doch an Größe. Daher hielten die Königinnen ihn für den König und fielen nach ihrer Sitte vor ihm nieder. Als hierauf einige von den gefangenen Eunuchen ihnen zeigten, wer Alexander sei, warf sich Sisygambis zu seinen Füßen und entschuldigte ihren Irrtum damit, dass sie den König nie zuvor gesehen hätten. Doch dieser richtete sie mit der Hand empor und sprach: ,Du hast dich nicht geirrt, Mutter, denn auch dieser ist Alexander (Curt. 3,12,15-17). ${ }^{3}$

Identität und Lebensdaten des Curtius sind unsicher, zumal das erste Buch mit der mutmaßlichen Selbstvorstellung des Historiographen zu den fehlenden Teilen der insgesamt zehn Bücher gehört. Gemäß aktueller communis opinio schrieb er als rhetorisch geschulter Angehöriger der Senatorenkreise im 1. J h. n. Chr., entweder unter Claudius oder unter Vespasian (PIR ${ }^{2}$ C 1618-19; Müller 2011; Atkinson 2009: v; Baynham 1998: 213-19). Seine Alexandergeschichte wird generell zur „Vulgata“ gezählt, eine inzwischen umstrittene Bezeichnung für die Quellen zur Alexandergeschichte, die auf dem Werk des Kleitarchos, Alexanders romanhaft schreibendem Zeitgenossen, als Hauptquelle basieren (Curt. 9.5.21; 9.8.15). In Curtius' Version der Zeltepisode liegt der Fokus sekundär auf der gnädigen Haltung Alexanders gegenüber den persischen Frauen und primär auf der Lobpreisung des freundschaftlichen Verhaltens gegenüber Hephaistion. Entsprechend seiner generellen moralisierenden Tendenzen lässt Curtius unmittelbar im Anschluss an den Bericht einen Exkurs zu Alexanders charakterlicher Entwicklung folgen:

Hätte er diese Bescheidenheit bis zum Ende seines Lebens beibehalten können, so wäre er meiner Meinung nach glücklicher gewesen als er zu sein schien, als er in ähnlichen Triumphzug wie Bacchus alle Länder vom Hellespont bis zum Okeanos siegreich durchmessen hatte. Dann hätte er sicherlich Stolz und Jähzorn, jene unbezwinglichen Laster, besiegt (...) Aber noch nicht hatte die Woge des Glücks seinen Sinn überwältigt, und darum trug er es zu Anfang mit Mäßigung und Weisheit, während er zuletzt seine Überfülle nicht zu fassen vermochte (Curt. 3,12,18-20).

Es ist davon auszugehen, dass die Episode in Curtius' Zeit längst zu einem schablonenhaften exemplum geworden war, das er benutzte, um sein Leitmotiv in den Historien, die Relation von fortuna und virtus in Alexanders Laufbahn, zu verfolgen (Bosworth 1980: 222). Von vorherrschenden Alexanderbildern in der römischen rhetorischen Tradition beeinflusst, entwarf er anhand einer Verfallsgeschichte des von seinen Siegen korrumpierten jungen Herrschers eine Studie zu politischer Macht und der Gefahr der Hybris (Müller 2011). Gerade die Bewertung von Alexanders generöser Haltung im Zelt der königlichen persischen Frauen dient Curtius dazu, eine Trennlinie zwischen dem tugendhaften, „guten“ makedonischen Herrscher Alexander vor seiner Korruption durch fortuna und dem späteren, zum topischen Klischee des dekadenten Perserkönigs

3 Zitiert nach der Übersetzung von J. Sibelis und H. Weismann in der Bearbeitung durch G. J ohns. 
entarteten „schlechten“ Alexander als eines neuen Xerxes aufzuzeigen (Atkinson 1980: 250). Hephaistion ist als Alter Ego des in der Zeltszene noch vorbildlichen Alexanders ebenfalls positiv konnotiert. Er ist sich zwar bewusst, dass er besondere Privilegien im Umgang mit Alexander genießt, nutzt diese aber nicht zur Überhöhung seiner eigenen Position aus. Wie sein Freund zeigt auch er sich (noch) bescheiden und maßvoll. Was sie indes unterscheidet, ist das Äußere. Curtius betont, dass Sisygambis Hephaistion mit ihm verwechselte, weil er, hochgewachsen und gut aussehend, ihren Vorstellungen von einem siegreichen Herrscher eher entsprach als Alexander. Interessanterweise schmälert diese Feststellung Alexanders Darstellung nicht. Durch seine Reaktion auf die Verwechslung erweist er sich als wahrhaft königlich, auch wenn er nicht so aussehen mag. Dagegen ändert sich Curtius' Ton, als er in späteren Passagen, nachdem der von ihm mit dem Gewinn der Entscheidungsschlacht angesetzte Negativwandel Alexanders bereits begonnen hat, abermals auf dessen eher unscheinbares Aussehen, seine kleine Statur und Hephaistions Anmut eingeht. Dies geschieht bei seiner Schilderung von Alexanders Begegnung mit der Amazonenkönigin Thalestris, die ein Kind von ihm möchte und von seinem wenig beeindruckenden Äußeren erstaunt ist (Curt. 6,5,29). Die unhistorische Episode der Begegnung mit der Amazone ist Teil der Schilderung von Alexanders ebenso rascher wie intensiver - hier - sexueller Depravation. In Curtius' Augen war Thalestris eine "Barbarin" (Baynham 1998: 170). Vorangegangen war zudem das Geschenk des Nabarzanes an Alexander in Gestalt des Eunuchen Bagoas, der einst ein Lustknabe Dareios' III. gewesen war und nun von Alexander in dieser Funktion übernommen wurde (Curt. 6,5,23). Die abermalige Erwähnung von Hephaistions anmutiger Schönheit erfolgt ebenfalls im Kontext von Curtius' moralisierenden Spitzen gegen Alexanders sexuelle Beziehungen: Mittels „pederastic phraseology“ (Ogden 2009: 210-11, A. 59) wird der einstmals vorbildliche Freund zum königlichen Lustknaben degradiert (Curt. 7,9,19).

Der griechische Historiograph Diodor, der im 1. Jh. v. Chr. eine Universalgeschichte verfasste, überliefert eine Version der Episode des Besuchs im persischen Zelt, die an eine gemeinsame Vorlage mit Curtius denken lässt (Atkinson 1980: 244, 253-54):

Als der Tag anbrach, nahm Alexander denjenigen seiner Freunde mit, der ihm am liebsten war, Hephaistion, und ging zu den Frauen. Sie waren beide gleich gekleidet, doch da Hephaistion hochgewachsener und schöner war, hielt Sisygambis ihn für den König und vollzog die Proskynese vor ihm. Als die anderen Anwesenden ihr Zeichen machten und mit der Hand auf Alexander zeigten, schämte sie sich für ihren Irrtum, machte aber einen neuen Ansatz und vollzog die Proskynese vor Alexander. Doch der König hielt sie auf, indem er sagte: ,Keine Sorge, Mutter, denn auch dieser hier ist Alexander (Diod. 17,37,5-6. Vgl. 17,114,2).

Diodor nennt den gleichen Grund für die Verwechslung wie Curtius, Hephaistions beeindruckende Erscheinung, und stellt auch die Großmut Alexanders und seine Loyalität zu seinem Freund heraus. 
Im Gegensatz zu Curtius und Diodor äußert Arrian leichte Zweifel an der Historizität der Begebenheit, weil sie nicht in seinen Hauptquellen, den Schriften der beiden Zugteilnehmer Ptolemaios und Aristobulos, überliefert wurde:

Darüber hinaus wird erzählt, dass Alexander selbst am nächsten Tag zusammen mit Hephaistion als einzigem seiner Freunde das Zelt betrat. Die Mutter des Dareios, die im Zweifel war, wer von beiden der König sei - beide trugen das gleiche Gewand -, sei vor Hephaistion getreten und vor diesem niedergefallen, denn dieser schien ihr der stattlichere. Als dieser nun zurückwich und jemand aus ihrer Umgebung auf Alexander zeigte, dies sei der König, habe sie sich ihres Irrtums geschämt und sich abgewandt. Doch Alexander meinte, sie habe sich keineswegs geirrt, denn auch dieser sei Alexander. Derartiges soll hier weder als verbürgte Wahrheit noch als völlig unglaubwürdig aufgezeichnet sein (Arr. an. 2,12,6-8).4

Arrian, der zu den typischen Vertretern der kulturellen Strömung der Zweiten Sophistik gezählt wird, die ihre Bildung als symbolisches Kapital zum gesellschaftlichen Aufstieg im Römischen Reich nutzten (Jones 1986: 149), schrieb im 2. J h. n. Chr. mit Xenophon als literarischem Vorbild eine Alexandergeschichte, die mit den kursierenden mythisierenden Verfremdungen Schluss machen sollte (Arr. an. 1,12,4-5). Er lobt die Ehre, die Alexander seinem Freund erwiesen hat, sollte es sich so zugetragen haben. Seine Zweifel an der Historizität der Episode teilt Arrian mit großen Teilen der Forschung, die von einer späteren anekdotenhaften Interpolation ausgehen (Badian 1998: 349; Stewart 1993: 71-2; Atkinson 1980: 252). In dem Verfasser wird meistens Kleitarchos vermutet, da er erstens ein besonderes Interesse am Schicksal der Sisygambis und zweitens an der Darstellung Hephaistions als Alexanders Alter Ego zeigte (Baynham 1998: 80; McKechnie 2005: 431).

Als entscheidend erscheint, dass Kallisthenes Alexanders Hofhistoriograph, der bis zu seiner Beseitigung 327 v. Chr. für den offiziellen Tatenbericht unter panhellenischen Vorzeichen zuständig war (Müller 2006: 280-81), wohl nicht als Quelle in Frage kommt (Atkinson 1980: 252). Es ist unwahrscheinlich, dass Alexander eine solche Geste, wenn sie sich denn zugetragen hatte, nicht der Öffentlichkeit hätte mitteilen lassen. So ist anzunehmen, dass die Episode eine Reflektion der späteren positiven Verklärung Alexanders, besonders als Philosophenschüler, gewesen ist. Die alternative These, es handle sich um eine Ableitung aus einer postumen Kultgemeinschaft Alexanders mit Hephaistion (Habicht 1956: 31-2), ist weniger wahrscheinlich, da es dafür bislang keine Belege gibt (Briant 2009: 136, A. 71, 169).

Alexanders Ausspruch, auch Hephaistion sei Alexander, rekurriert auf die Freundschaftslehre seines Lehrers Aristoteles und zeigt Alexander im Licht des Idealimages des Philosophen auf dem Thron. Gemäß der aristotelischen Theorie war ein Freund eine Seele in zwei Körpern, repräsentierte das andere Ich und erkannte seine guten

${ }^{4}$ Zitiert nach der Übersetzung von G. Wirth. 
Eigenschaften im anderen (Aristot. Eth. Nic. 1156 B, 1157 B, 1159 B; Diog. Laert. 5,20; Plut. moral. 93 E. Vgl. Konstan 1997: 42, 75). Davon zu unterscheiden war der Schmeichler, der wie ein Schatten folgte und anbiedernd allem zustimmte (Plut. moral. 53 B).

Darüber, dass Hephaistion Alexanders engster Freund war, herrscht in den Quellen Einigkeit (Diod. 17,114,1; Nep. 18,2,2; Curt. 3,12,15-16; Plut. Alex. 39,5; mor. 339 F-340 A; 180 D; 332 F-333 A). Dagegen sind explizite Belege dafür, dass diese Freundschaft eine Liebesbeziehung war, weniger zahlreich und teils von wesentlich späteren Quellen überliefert (Luk. Calum. 17; dial. mort. 12,4; Ael. VH 12,7; Epikt. 2,22,17; Diog. Epist. 24,1 Hercher), deren Glaubwürdigkeit sehr kritisch zu sehen ist (Ogden 2009: 211-2). Hephaistion wird darin als eromenos Alexanders gekennzeichnet, der mittels seiner sexuellen Anziehungskraft Einfluss auf den Herrscher ausübte. Unter den Alexanderhistoriographen sind es Curtius und Justin, die zumindest auf eine erotische Beziehung anspielen (J ust. 12,12,11-12; Curt. 7,9,19).

Als Indiz gilt überdies die Gleichsetzung von Hephaistion mit Patroklos, dem Alter Ego von Achilles (Plut. mor. 93 E), Alexanders Urahnen mütterlicherseits (Ameling 1988: 667; Reames-Zimmerman 1998: 242; Stewart 1993: 83). Obwohl in der Ilias nicht explizit gesagt wird, dass Achilles und Patroklos ein Liebespaar waren, gingen Alexanders Zeitgenossen in der griechisch-makedonischen Welt des 4. J h. v. Chr. davon aus, dass es sich um eine solche Beziehung gehandelt habe (Aischin. 1,133.142-144; Plat. Symp. 179 E180 B; Arr. Per. 23,4). Dies sei für die Zuhörer des Epos so selbstverständlich gewesen, dass sich konkrete Hinweise erübrigt hätten (Konstan 1997: 37; Halperin 1990: 86-7). Wenn sich Alexander und Hephaistion daher der Öffentlichkeit als Achilles und Patroklos präsentiert oder diesen Vergleich zumindest nicht dementiert hatten, wäre dies eine deutliche Stellungnahme zum Charakter ihrer Beziehung gewesen. Indes lässt sich eine Datierung dieser Parallele in Alexanders Lebzeiten nicht nachweisen. Obwohl der überwiegende Teil der Forschung nicht daran zweifelt, dass Hephaistion Alexanders Lebensgefährte war (Reames-Zimmerman 1999: 92; Heckel 1993: 65-6, Berve 1926: 173; Wirth 1967: 1022) - eine Beziehung, die durchaus innerhalb der Normen der makedonischen höfischen Kultur seiner Zeit gewesen wäre (Ogden 2009: 213, 217) - , wird aktuell davon ausgegangen, dass nicht nur Hephaistions Angleichung an Patroklos, sondern auch Alexanders Achillesimitatio eine spätere literarische Ausschmückung war (Heckel 2011): Alexander selbst habe höchstens seine Abkunft von Achilles betont, etwa mittels der Angleichung seines Porträts an die Ikonographie seines Urahns (Hölscher 2009: 30-32).

Als ein späterer Urheber der Parallele zum homerischen Heldenpaar lässt sich Arrian ausmachen, der, vielleicht mit dem römischen Beispiel von Hadrian und Antinoos vor Augen (Stadter 1980: 39, 169), dem Motiv eine nachhaltige Wirkung verlieh. So gibt er eine Tradition wieder, wonach Hephaistion der Öffentlichkeit bereits zu Beginn des Persienfeldzugs als der neue Patroklos des makedonischen Achilles präsentiert wurde. In 
Ilion soll er, parallel zur Bekränzung von Achilles' Grab durch Alexander, einen Kranz auf Patroklos' Grab gelegt haben (Arr. an. 1,12,1; vgl. Ael. VH 12,7). ${ }^{5}$ Die Historizität der Episode ist stark umstritten. Zwar hätte die öffentliche Doppelgeste durchaus in Alexanders panhellenische Selbstdarstellung zu Beginn des Zugs gepasst. Doch gibt zu denken, dass abermals ausgerechnet Kallisthenes die parallele Grabbekränzung ebenso wie den gemeinsamen Besuch bei den Achaimenidinnen anscheinend nicht überliefert hat (Heckel 1992: 67). Daher ist anzunehmen, dass es sich um eine spätere Erfindung handelt (Badian 1998: 350; Bosworth 1980: 221), die wiederum teilweise auf einen gemeinsamen postumen Kult mit Hephaistion als paredros Alexanders zurückgeführt wird (Habicht 1956: 32; Heckel 1978: 226), aber auch aus dem Doppelgängermotiv resultiert oder dazu gedient haben kann, ihre Beziehung als erotisch zu charakterisieren.

Die Stilisierung Hephaistions zum Patroklos von Achilles-Alexander wird vor allem in den Berichten zu Alexanders Reaktion auf seinen Tod manifest (Plut. Alex. 72,2-3; Arr. an. 7,14,4-6; Ael. VH 7,8; Diod. 17,110,8), die gerade deswegen kritisch zu betrachten sind. Alexanders - sicherlich nicht zu bezweifelnde - Verzweiflung über den politisch wie menschlich bedeutsamen Verlust und die von ihm aufwändig ausgerichteten Trauerfeierlichkeiten (Arr. an. 7,14,8-9; Diod. 17,110,8. 114,1; Plut. Alex. 72,3-4; Ael. VH 7,8; Just. 12,12,12) werden literarisch an die homerischen Schilderungen von Achilles' Schmerz und über die Verbrennungszeremonie von Patroklos' Leichnam angeglichen (Il. 23,141-150). Auch Alexanders folgender Winterfeldzug gegen die Ethnie der Kossaier wird aufgrund des grausamen Vorgehens mit Achilles' Totenopfern in Gestalt der zwölf jungen Trojaner für Patroklos' Scheiterhaufen parallelisiert (Plut. Alex. 72,3; Il. 23,175-177), vermutlich zuerst wiederum durch Kleitarchos (Hammond 1993: 298). Zudem wird über den Plan eines großen Funeralmonuments für Hephaistion berichtet, dessen Ikonographie teilweise der von Alexanders Leichenwagen ähnelte (Diod. 18,4,2. Vgl. Palagia 2000: 169172).

In der antiken Tradition ist Hephaistion als Alter Ego Alexanders konsequent an dessen charakterliche Entwicklung und sein Schicksal angeglichen. Hephaistions Aufstieg ist untrennbar mit Alexanders Machtgewinn verbunden, auch ausgedrückt in der Anekdote, Alexander habe ihm bei einem ihrer seltenen Streits mitgeteilt, ohne ihn sei er ein Nichts (Plut. Alex. 47,6; mor. 337 A). Von Bedeutung erscheint, wie sehr diese Stilisierung von Hephaistion als Alexanders Doppelgänger das Porträt seiner Karriere prägt. Während er zu Beginn als Aristotelesschüler figuriert und keine Negativtradition über ihn fassbar ist, ändert sich dies auffälligerweise parallel zu Alexanders Negativentwicklung. Je mehr dessen Wandel zum autokratischem, den makedonischen Traditionen entfremdetem König von Asien voranschreitet, desto intriganter, anmaßender und schmeichlerischer gegenüber Alexander erscheint auch Hephaistion. Wie bei seinem Freund sind auch bei ihm ein moralischer Verfall und Ansätze zur Hybris festzustellen.

5 J ust. 11,5,12; Diod. 17,17,3 und Plut. Alex. 15,4-5 erwähnen nur den Besuch Alexanders an Achilles' Grabstätte. 
Beide lassen von ihrem einstigen Status als Philosophenschüler kaum noch etwas erkennen.

Hephaistion gilt als derjenige, der Alexanders Persienpolitik und die Adaption achaimenidischer Hofetikette vorbehaltlos und am engagiertesten unterstützte, was ihm teils in der antiken Tradition nicht unbedingt positiv angerechnet wird (Plut. Alex. 47,5). Er soll ihm bei dem gescheiterten Versuch, die Proskynese auch für die Makedonen bei Hof einzuführen, und bei dem Sturz missliebig gewordener Regimekritiker - Philotas und Kallisthenes - nachgeholfen haben (Curt. 6,11,10; Plut. Alex. 55,1). Im Zuge seines Aufstiegs wurde er hochfahrend und arrogant, geriet mit anderen hochrangigen Amtsträgern in Kompetenzfragen aneinander (Plut. Alex. 47,5-6; Eum. 2,1-4; Arr. an. 7,13,1. 14,9) und soll sogar mit Alexanders Mutter Olympias gestritten haben: Ein in seiner Historizität sehr fragwürdiger Brief Hephaistions an sie ist in einem hochfahrenden Ton gehalten und impliziert, dass er für sich selbst königsgleich den Pluralis Majestatis verwendete (Diod. 17,114,3).

Entsprechend dominiert in der Forschung das Bild Hephaistions als eines unangenehmen Günstlings, dessen einzige Qualifikationen sein gutes Aussehen und die kriecherisch folgsame Hingabe gegenüber dem Herrscher gewesen seien und der im Zuge seines unverdienten Aufstiegs rasch die Bodenhaftung verloren habe (Badian 1998: 350; Heckel 1993: 83; Carney 1975: 221; Berve 1926: 173). Dagegen ist allerdings einzuwenden, dass dieser Eindruck von seiner antiken Stilisierung zu Alexanders Doppelgänger, der einen spiegelbildlichen moralischen Verfall vollzog, herrührt. In Anbetracht des literarischen Charakters des Motivs ist diese Darstellung Hephaistions sehr kritisch zu betrachten und entsprechend als topisch zu bewerten. Gegen seine Porträtierung als Alexanders zweites, entartetes Ich sprechen auch die Zeugnisse, die davon berichten, dass Hephaistion häufig für diplomatische Missionen und Städtegründungen eingesetzt wurde (Curt. 4,1,20; 8,12,6; Diod. 17,47,1-6. 91,1-2; Arr. an. 5,20,6; 21,2-6; Plut. Alex. 47,5) sich im administrativen und organisatorischen Bereich bewährte (Müller 2003: 227; Heckel 1993: 69; Reames-Zimmerman 1998: 100-124) und auch unter den Makedonen keinesfalls isoliert war (Plut. Alex. 47,6). Gerade als Diplomat benötigte er Fingerspitzengefühl und konnte sich ein Verhalten, wie es die Quellen teils schildern, sicher nicht leisten.

\section{DER TOD DES DOPPELGÄNGERS}

Wie stark das Doppelgängermotiv in der antiken Literatur auf Hephaistion und Alexander angewendet wurde, zeigt sich insbesondere in den mit Skepsis zu behandelnden Berichten über die Zeit nach Hephaistions Tod. Die Maßnahmen, die Alexander zu Ehren und Gedenken des Verstorbenen getroffen haben soll, besaßen einen königgleichen Charakter und betonten, dass mit ihm der zweite Alexander gestorben war. So soll Alexander die Hinrichtung des Arztes, der Hephaistion vor seinem Tod behandelt und nicht gerettet hatte, befohlen haben, vermutlich durch Kreuzigung (Plut. Alex. 72,2; Arr. 
an. 7,14,4) - 6 eine Hinrichtungsart, mit der auch Königsmörder bestraft wurden (Curt. 7,5,40-41; J ust. 9,7,10; 12,5,11). Der kostspielige Scheiterhaufen, auf dem Hephaistions Leichnam mit seinen Waffen, Preziosen und Porträtskulpturen der anderen Hetairen als Opfergaben in einer Zeremonie, die den Bestattungen makedonischer Herrscher entsprach (Palagia 2000: 168), verbrannt worden sein soll (Arr. an. 7,14,8-9; Diod. 17,114,1. 115,1; Plut. Eum. 2,4-5; Ael. VH 7,8), verweist auf seine besondere Stellung im Reich. Noch deutlicher zeigt dies Alexanders - in seiner Historizität umstrittener (Brosius 2003: 181) Befehl, das heilige Feuer zu seinem Gedenken löschen zu lassen, eine Ehre, die im Perserreich nur dem toten König zustand (Diod. 17,114,4). Alexanders Alter Ego konnte nicht ersetzt werden; seine Kommandantur der Hetairenreiterei blieb vakant (Arr. an. 7,14,9-10), eventuell auch sein Leibwächterposten.

Sicherlich begünstigt durch den Umstand, dass Alexander nur wenige Monate nach ihm starb, wurde der Deutung entsprechend, dass der Tod des Doubles nicht ohne den eigenen Tod abgeht, Hephaistions Ableben zum Vorzeichen für Alexanders Ende stilisiert: „Hephaistion is also Alexander: Hephaistion's funeral is also an omen of Alexander's death“ (McKechnie 1995: 419). Besonders das Löschen der Königsfeuer wurde als schlechtes Omen dargestellt und in der literarischen Überlieferung durch weitere üble Vorzeichen im Vorfeld von Alexanders Rückkehr nach Babylon ergänzt (Diod. 17,112,2. 114,4. 116,2-4; Arr. an. 7,18. 22,2-5). Nicht umsonst erinnert wohl Diodor, als er über Hephaistions Bestattung und die schlechten Omina bezüglich Alexanders nahem Ende schreibt, noch einmal an die symbolische Szene mit Sisygambis und daran, wie Alexander Hephaistion öffentlich als sein anderes Ich propagiert hatte (Diod. 17,114,2). Als seine Quelle wird Kleitarchos, der "Spezialist" für das Alter Ego-Motiv, vermutet (McKechnie 2005: 420, 431).

Das Bild wird abgerundet, wenn man die wenigen Fragmente und den Titel einer pamphletartigen Schrift des Ephippos von Olynth ansieht, in den Varianten Über den Tod von Alexander und Hephaistion oder Über das Begräbnis von Alexander und Hephaistion überliefert (FGrHist 126, F 1-3). Ephippos, der eine recht makedonenfeindliche Attitüde an den Tag legte und Alexanders Hofleben in den dunkelsten Farben schilderte, schrieb beide Todesfälle ihrem unmäßigen Alkoholmissbrauch bei den makedonischen Symposien zu (Athen. 3,120 D-E; 10,434 A-B). Paul McKechnie vermutet, das Doppelgängermotiv habe auch bei ihm bereits im Vordergrund gestanden: „In preparing to bury Hephaestion, Alexander was unwittingly preparing to die himself (Hephaestion was also Alexander)“ (McKechnie 1995: 430).

\footnotetext{
${ }^{6}$ Der von Arrian gebrauchte Terminus kann für „hängen“ und für „kreuzigen“ stehen.
} 


\section{DER DOPPELTE ALEXANDER IN DER KUNST:}

\section{IL SODOMA, VERONESE, LEBRUN}

In der europäischen Kunst der Renaissance und des Barock wird das Motiv von Hephaistion als Alter Ego Alexanders in der Konnotation des treuen Waffenkameraden wieder aufgegriffen und sogar visuell erweitert: Hephaistion gerät zum identisch aussehenden Doppelgänger des Makedonenherrschers.

Während die mittelalterliche Alexanderrezeption von Ambivalenz geprägt war und neben dem fürstlich-höfischen Musterbild auch das abschreckende Exempel von superbia in seiner Figur verkörpert sah (Noll 2005: 10-28; Stoneman 2008: 90-169), dominierte in der Renaissance das Bild Alexanders als Prototyp des jugendlichen Feldherrn, tapferen Kriegerkönigs und heldenhaften Eroberers (Starn 1986; Schwarzenberg 1969: 403). Die Renaissancekünstler stellten Alexander jedoch nicht nur als Krieger dar. Seine Funktion als Modell fürstlicher Tugenden, die durch verschiedene Episoden der Alexandergeschichte als standardisierten exempla versinnbildlicht wurden (Krause 1997: 223), umfasste auch das Bild des höfischen Kavaliers und galanten Frauenliebhabers, das in der Antike gar nicht und in den westlichen Alexanderlegenden des Mittelalters von untergeordneter Bedeutung gewesen war (Wilson-Chevalier 1997: 27-31).

Bei der Rezeption der antiken Ikonographie zur Gestaltung des Alexanderbilds führte allerdings eine irrtümliche Deutung dazu, dass, insbesondere in Italien und Frankreich, ein Typus dominierte, der einen recht femininen Alexander zeigt: jugendlich bartlos, mit Kürass und korinthischem Helm, schulterlangen Locken und weichen, androgynen Gesichtszügen (Baynham 2009: 295). Ein so gestalteter Alexander erscheint ohne große Variationen in Plastik und Malerei etwa bei Andrea del Verocchio, Il Sodoma, Pietro da Cortona und Charles LeBrun und bleibt auch in der weiteren Entwicklung der Alexanderdarstellung in der europäischen Kunst einflussreich.

Der Typus leitet sich von dem Kopf ab, der auf dem Avers von Alexanders Goldstateren zu sehen ist und die Göttin Athena darstellt. Zum folgenschweren Missverständnis kam es, als die Gelehrten der Renaissance von der Legende auf dem Revers, Alexandrou, darauf schlossen, dass sein Porträt auf der Münze gezeigt sein müsse, während die Beischrift tatsächlich nur dazu diente, den Makedonenherrscher als Prägeherrn zu kennzeichnen. Da neben der Legende eine geflügelte Nike dargestellt war, wurde im Ausschlussverfahren angenommen, der Kopf auf dem Avers müsse Alexanders Porträt sein (Kraft 1965: 11). Der Händler und Antiquar Cyriacus von Ancona, der zwischen 1412-1452 in der Ägäis Informationen zur Antike sammelte und bei humanistischen Gelehrten und Künstlern als wissenschaftliche Autorität galt, soll einer der Urheber des Missverständnisses gewesen sein (Vickers 1999: 29-30). Somit wurde Alexander unfreiwillig zu einem Doppelgänger der Athena.

Ein solcher Athena-Alexander ist auch auf dem Freskenzyklus des italienischen Malers Antonio Giovanni Bazzi, genannt Il Sodoma (1477-1549), in der Villa Farnesina in Rom zu sehen. Im Auftrag des Sieneser Bankiers und Händlers Agostino Chigi schuf er um 1516 
eine Dekoration, die Alexander als Vertreter der hohen Liebe zeigte (Wilson-Chevalier 1997: 29) und darauf hinkonzipiert war, die umstrittene Hochzeit seines Auftraggebers zu legitimieren (Noll 2005: 36; J ones/ Penny 1983: 184, 338). Gezeigt wurden die Hochzeit Alexanders mit der baktrischen Adligen Roxane und die Familie des Dareios vor Alexander.

Literarische Grundlage für die Zeltszene war Curtius' Alexandergeschichte (Noll 2005: 32), die seit dem Mittelalter beliebt war, die Entstehung von Alexanderromanen beeinflusste und deren editio princeps um 1470 in Venedig entstand (Baynham 1998: 4). $\mathrm{Zu}$ sehen ist vor der Kulisse des Zelts Sisygambis, die, umgeben von Frauen und Kindern, einen Kniefall vollführt. Vor ihr stehen zwei identisch gewandete Krieger mit Rüstung, Mantel und korinthischem Helm. Sie sind von gleicher Größe, tragen die gleiche Frisur und ähneln sich wie Zwillinge. Der rechte Mann, vor dem sie sich niedergeworfen hat, steht mit dem Rücken zum Betrachter, ins Halbprofil gekehrt, und hat die linke Hand in die Hüfte gestützt. Sein Freund zu seiner Rechten neigt sich in einer beschwichtigenden Geste der Großmut zur persischen Königinmutter hinab, die anscheinend gerade ihren Irrtum bemerkt hat, und will sie aufrichten. In Abwandlung zu Curtius wird anhand dieses Freskos deutlich, wie es zu der Verwechslung kommen konnte, da Alexander und sein Alter Ego Hephaistion sich so verblüffend ähnlich sehen.

Auch Alexanders Hochzeit mit Roxane, basierend auf Lukians spöttischer Ekphrasis eines - vielleicht fiktiven - Gemäldes des Malers Aëtion (Luk. Hdt. sive Aet. 4-7), ${ }^{7}$ stellt das Freundespaar mit ähnlich welligen, langen, hellen Haaren und jugendlichen, androgynen Zügen dar. Während Alexander seiner Braut Roxane eine Krone darbietet, sieht Hephaistion als Fackelträger und Begleiter des Bräutigams zu, mit einer Hand auf die Schulter des Hochzeitsgotts Hymeneus gestützt (Noll 2005: 33; Wilson-Chevalier 1997: 29).

Der venezianische Maler Paolo Veronese behandelte das Motiv des gemeinsamen Besuchs der persischen Königsfamilie ebenfalls in einem Gemälde für Francesco Pisani für den Palazzo Pisani in Montagnana (Terribile 2009; Cocke 1978). Um die Mitte der 1560er Jahre schuf er dabei einen Alexander, der abweichend von dem von Il Sodoma aufgegriffenen Typus maskuliner aussah, mit kurzem dunklen Haar und einem schmalen Backenbärtchen. Wiederum erscheint Hephaistion indes als sein zum Verwechseln ähnlicher Doppelgänger. Die Betonung von Alexanders Großmut gegenüber dem Freund gilt bei Veronese als ausgeprägter als bei Sodoma, der die Milde und Galanterie des Siegers in den Vordergrund rückte (Cocke 1978: 325-26).

Der französische Maler Charles LeBrun, der die Jahre 1642-1645 in Rom verbracht hatte, kannte die italienischen Vorgaben und adaptierte die dort vorherrschende Athena-

${ }^{7}$ Der syrische Satiriker, der als einziger ein solches Bild erwähnt, scheint in dieser Passage seiner Spottschrift das tatsächliche Gemälde Aëtions einer Hochzeit zwischen Semiramis und Ninos (Plin. NH 35,78 zu parodieren und sich über Alexanders größere Vorliebe für seinen Brautführer Hephaistion (Stewart 2003, 41) lustig zu machen. 
Alexanderikonographie für sein 1661/62 entstandenes Gemälde Alexander vor der Familie des Dareios (Grell/Michel 1988: 107; J affé 1963: 558). 1661 hatte er von Ludwig XIV. kurz nach dessen Antritt der Alleinherrschaft den Auftrag erhalten, die königliche Identifikationsfigur Alexander (Schmitter 2002: 420-21; Hadjinicolaou 1997: 20) in einem Sujet seiner Wahl zu malen (Schwarzenberg 1969: 403; Posner 1959: 239). Welche Symbolik er in der Episode um die persischen königlichen Frauen sah, illustriert die Legende eines von ihm autorisierten Kupferstichs des Gemäldes: „Il est d'un Roy de se vaincre soy-mesme“ (Posner 1959: 241). Mit diesem ersten Teil seines bis 1672/3 vollendeten Alexanderzyklus' (Kirchner 2001; Birkenholz 2002) für die Dekoration von Versailles begründete LeBrun seine Stellung am Hof als premier peintre du Roi (Schmitter 2002: 399). ${ }^{8}$

Wie bei Sodoma sind Hephaistion und Alexander fast zwillingsgleich dargestellt, tragen jedoch im Widerspruch zu den antiken Quellenberichten unterschiedliche Kleidung: Hephaistion einen roten Mantel mit einer Kamee, die Alexanders Porträt zeigt, und einen dunklen Brustpanzer mit Goldornamenten. Alexanders Mantel ist beige-golden, der Brustpanzer silbern mit Goldornamenten (Baynham 2009: 307). Das Licht, das auf sein Gesicht fällt, während Hephaistion im Schatten steht, und die weit ausholende Gestik mit dem Pathos der Milde signalisieren dem Betrachter jedoch, wer der Herrscher ist.

Auch in der weiteren Entwicklung der Alexanderdarstellung in der europäischen Kunst blieb das Motiv der äußerlichen Ähnlichkeit zwischen Alexander und Hephaistion weit verbreitet.

\section{ALEXANDER UND HEPHAISTION IN BIOGRAPHIEN DES 20. JAHRHUNDERTS}

Im 20. J ahrhundert war die ambivalente Faszination, die von der schon in der Antike schablonenhaft gewordenen Projektionsfigur des Eroberers und Weltherrschers ausging, ungebrochen. Zeitpolitische Strömungen beeinflussten das Urteil über seine Person und Leistungen, so dass teilweise Alexanderbilder entstanden, die deutlich mehr die Ereignisse und kulturellen Strömungen des 20. Jahrhunderts reflektierten als Erkenntnisse zum makedonischen Reich des 4. Jahrhunderts v. Chr. Dieses Phänomen findet sich sowohl in der wissenschaftlichen als auch in der nicht-wissenschaftlichen Literatur zu Alexander.

Im Folgenden werden exemplarisch zwei Alexanderbiographien behandelt, die aus dem Bereich der Fachwissenschaft stammen, aber für einen breiten Leserkreis mit populärwissenschaftlichem Ansatz geschrieben sind, zudem ein jeweils sehr persönliches Alexanderbild widerspiegeln und als einflussreich gelten können: Alexander der Große. Das Problem seiner Persönlichkeit und seines Wirkens des österreichischen Althistorikers

81719 merkte der Abbé Du Bos in seinen Réflexions critiques sur la poésie et sur la peinture jedoch an, dass LeBrun für das Alexanderporträt irrtümlich eine Darstellung von Minerva-Athena auf einer Münze Alexanders verwendet habe. Daher sehe er nun schön wie eine Frau aus (Grell/Michel 1983: 112). 
Fritz Schachermeyr aus dem J ahr 1973 sowie die Alexanderbiographie von Robin Lane Fox aus dem Jahr 1974, die nicht zuletzt deswegen 2004 wieder Relevanz gewann und neu aufgelegt wurde, weil er als historischer Berater für Oliver Stones Film Alexander tätig war (Müller 2005).

Bei Schachermeyrs Werk handelt es sich um eine revidierte Fassung seiner Alexanderbiographie aus dem J ahr 1949. In beiden Versionen entsteht ein ambivalentes, von politischen Zeitbezügen und moralischer Wertung geprägtes Bild Alexanders als eines über menschliche Maßstäbe hinausgehenden Genies und Titanen, der in einem als dämonisch charakterisierten Kraftakt der Welt seine Ordnung aufzwang (Wirth 1985).

Der zum politischen Symbol geratene Alexander wird als unzertrennlich mit seinem Jugendfreund Hephaistion beschrieben (Schachermeyr 1973: 144), der in der Rolle als sein Patroklos erscheint. So hält Schachermeyr auch die Episode mit der parallelen Bekränzung der Heldengräber in Ilion für historisch (Schachermeyr 1973: 164). Das Motiv des Alter Egos wird indes keinesfalls nur positiv bewertet, sondern läuft auf eine ungute Verquickung von privaten Emotionen und machtpolitischen Strukturen hinaus, die Nepotentum und Willkür nach sich zieht. Schachermeyr beschreibt Hephaistion folgendermaßen:

Dieser, ein Mann nun von seltener Schönheit, seit neuestem den orientalischen Sitten besonders geneigt, zeigte sich wie kein anderer von des Königs Plänen begeistert. Begabt zwar als Organisator und Feldherr, stand er dem Herrscher doch vor allem rein menschlich nahe. Das Sentimentale, bei Alexander viel ausgeprägter als bei anderen Großen der Geschichte, offenbarte sich in nichts stärker als in diesem fast weichlichen Verhältnis höriger Herzen (Schachermeyr 1973: 327).

Schachermeyrs Definition dieses Alter Egos hat wenig mit der aristotelischen Idealvorstellung eines Freundes zu tun, sondern vielmehr mit Plutarchs abfälliger Schilderung des Schmeichlers. Hephaistion machte sich demnach bei Alexander primär deswegen unentbehrlich, weil er ihn niemals kritisierte. Schachermeyr konstatiert daher als Grund für seinen Aufstieg eine

fast weibisch schmeichelnde Hörigkeit, die der Herrscher an seinem Liebling schätzte und die er zärtlich vergalt (...) Jedenfalls wurde der Günstling so sehr zum schmiegsamen Schatten, zum alter ego des Herrschers, dass er von ihm in bedeutsamer Stunde zu sagen wusste: Auch er wäre Alexander. (Schachermeyr 1973: 511).

Diese Vorstellung eines Alter Egos als eines kriecherischen Opportunisten entspricht nicht dem Konzept, wie es die antiken Quellen der Zeltszene zugrunde legen. Bei Schachermeyr resultiert die Negativdarstellung aus seiner Schilderung Hephaistions als eines neidischen, eifersüchtigen Favoriten, der alles tat, um der einflussreichste Mann nach dem König an Alexanders Hof zu werden. Um dieses Ziel zu erreichen, ging er intrigant und berechnend gegen seine Konkurrenz vor und buckelte vor dem Herrscher (Schachermeyr 1973: 511-12). Der Umstand, dass Alexander einen derart gewissenlosen 
Opportunisten förderte, trägt zu seinem ambivalent gestalteten Image bei: loyal zu seinen Jugendfreunden, aber teils fragwürdig in seinen personalpolitischen Entscheidungen und zu sehr von seinen Empfindungen gesteuert.

Der britische Althistoriker Robin Lane Fox entwirft dagegen ein überaus positives Bild Alexanders. Bei ihm gerät er zu einem in der makedonischen Adelsetikette verwurzelten jugendlich idealistischen Entdecker und Kämpfer. Lane Fox orientiert sich dabei stark an den Vorgaben seines Landsmanns William Woodthorpe Tarn (Badian 1976: 229), der 1948 die hagiographisch anmutende Fiktion eines sakrosankten Weltverbesserers und Gentleman entworfen hatte, der, erfüllt von strahlendem Idealismus, seine Feldzüge vor allem mit dem Ziel führte, sein Ideal einer Menschheitsverbrüderung verwirklichen zu können (Müller 2003: 8).

In Lane Fox' von der Forschung stark kritisierten Biographie - "an adventure story mid-way between historical journalism and historical fiction" (Badian 1976: 230) - wird das Verhältnis zwischen Alexander und Hephaistion als eine innig vertraute Liebesbeziehung beschrieben, die lebenslang hielt. Sie ergänzten sich dabei so perfekt, dass sie einander beflügelten und nur zum Besten inspirierten: „aus Hephaistion wurde der überaus fähige Anführer der Kavallerie Alexanders und sein Vizekönig, ehe er als ein göttlicher Held starb, dem postume Verehrung gebührte“ (Lane Fox 1974: 72).

Dieser verklärten Stilisierung der beiden zum heroischen Paar entsprechend hält Lane Fox den Vergleich mit Achilles und Patroklos für zeitgenössisch und bewertet die Bekränzung der Heldengräber und die Szene im Zelt der Achaimenidinnen als historisch (Lane Fox 1974: 146, 228-29). Die innere Verbundenheit zwischen Alexander und seinem Alter Ego wird als so stark und unverbrüchlich dargestellt, dass der Tod Hephaistions zwingend auch für Alexander zum Tod führt: „Doch wie sehr er sich auch gegen den Verlust Hephaistions wappnen mochte, die Orakel hatten schon angedeutet, dass er ihn nie überwinden werde" (Lane Fox 1974: 596).

\section{ALEXANDER UND HEPHAISTION IN DER POPULÄRKULTUR DES 20. UND 21. JAHRHUNDERTS: KLAUS MANN, ROGER PEYREFITTE, OLIVER STONE}

In Klaus Manns zu seiner Zeit zwiespältig aufgenommenen Prosawerk Alexander, Roman einer Utopie von 1929/30 (Naumann 2001: 93), in dem er „in Alexander einen Homosexuellen zeigt, der letztendlich an seiner Liebe und seiner Einsamkeit scheitert" (Zynda 1986: 71), wird die Darstellung Hephaistions als sein Doppelgänger einer umfassenden Revision unterzogen. Aus der traditionellen Vorgabe einer innigen, harmonischen Verbundenheit wird eine Entfremdung vom Alter Ego in Gestalt einer verkrampften, unglücklichen, zunehmend distanzierten Beziehung, die immer in der Schwebe bleibt und keine Erfüllung findet.

Alexander, anfangs als freundlich und fröhlich charakterisiert, ein gebildeter,

sensibler, aber etwas labiler Herrscher, der Gewalt in einem „panerotischen 
Wunschtraum“ (Naumann 2001: 92) durch Liebe und Verständnis ersetzen will (Härle 1988: 299), wandelt sich durch seine Siege und unerwiderten Gefühle für den narzisstischen Kleitos zum unnahbaren, schroffen, despotischen Machtmenschen (Heißerer 2006: 231; Härle 1988: 300-301, 379, Anm. 2; Zynda 1986: 70). Im Unterschied zur antiken Tradition, wonach Hephaistion bis zuletzt loyal zu Alexander hielt und auch dessen heftig umstrittene Persienpolitik unterstützte (Plut. Alex. 47,5), zeichnet Klaus Mann eine schleichende Zerrüttung ihrer Freundschaft durch den Negativeffekt des Erfolgs auf Alexander nach. Die Entfremdung deutet sich schon auf dem Weg zum Hellespont an, als Alexander eine missglückte Annäherung an Hephaistion wagt: „Er verstummte, denn schon merkte er, dass der andere nichts begriff. Er merkte, dass er, wie nur je, alleine war. Einsamkeit machte ihn demütig, nicht mehr stolz; er suchte dem näher zu kommen, der fremd neben ihm stand“ (Mann 2006: 60).

Beide werden in einer Inversion als Gegensatzpaar dargestellt, sowohl äußerlich als auch innerlich. Alexander hat stählerne graue Augen und schlangenhaftes Haar. Er ist „heller, muskulöser und elastischer" als Hephaistion mit seinem ernsten, schönen Gesicht und „feierlich guten Blick“ (Mann 2006: 19). Er wirkt „bräunlich, weicher und mit einer Neigung zur lieblichen Schwerfälligkeit“ (Mann 2006: 19, 63). Ihre Freundschaft erscheint nicht als eine Partnerschaft von zwei Gleichberechtigten, sondern in deutlicher Hierarchisierung als Beziehung zwischen Herrscher und - wenn auch privilegiertem Untergebenen. Alexander, begabt und energisch, entwickelt sich zum unerbittlichen Tyrannen. Die Fassade droht zwar einige Male zu bröckeln, wird von ihm jedoch stets mit noch strengerer Härte bewahrt. Auch wenn er auf den ersten Blick als der Stärkere figuriert und Hephaistion nicht wie ein ebenbürtiger Waffengefährte, sondern mehr wie ein folgsames Hündchen erscheint, ist er doch nur der vermeintlich Schwächere: Er beweist Charakterfestigkeit, lässt sich von seinem Aufstieg nicht verbiegen und bleibt sich bis zu seinem Ende selbst treu: unverändert verständnisvoll, geduldig, rücksichtsvoll, bescheiden, zurückhaltend, verlässlich, loyal und freundlich.

Der Darstellung entsprechend, dass Alexander gegen die Verbundenheit mit Hephaistion eher ankämpft als sie sucht, wird die Parallele zum epischen Paar Achilles und Patroklos nicht von ihm selbst propagiert, sondern von außen, durch ihre makedonischen Kameraden, an sie herangetragen. Der Vergleich ruft prompt Alexanders Erstaunen hervor (Mann 2006: 63).

Die wiederkehrende Behauptung, dass Hephaistion am Hof als sein innigster Vertrauter gilt, wirkt angesichts seines „standhaft innigen, verzichtenden und aussichtslosen Werbens um Alexander, der immer unnahbarer, immer unbegreiflicher sich mitten in seiner Einsamkeit befand“ (Mann 2006: 127) wie eine ungerechtfertigte Außenprojektion. Konsequent ist daher auch die Szene, in der Alexander sich einmal auf die Alter Ego-Vorstellung einlässt, als eine Maskerade in alberner Laune geschildert. Während seines Indienfeldzugs schlüpft er in Hephaistions Rolle, um einen indischen Prinzen zum Narren zu halten. Sein Leibwächter Ptolemaios dagegen spielt Alexander. Es handelt sich um eine Episode aus der Kompilation fabulöser Abenteuergeschichten um 
Alexander, dem antiken Alexanderroman. Darin verkleidet Alexander Ptolemaios ebenfalls mit seiner Königstracht, als Prinz Kandaules vorspricht, und gibt sich später bei seinem Besuch bei dessen Mutter, Königin Kandake, als sein Gesandter Antigonos aus, vermag sie jedoch nicht über seine Identität zu täuschen (Ps.-Kall. 3,19-22).

Beunruhigt beobachtet vom echten Hephaistion, imitiert Alexander dessen bescheidene, sanfte Freundlichkeit und „ließ sich von allen Hephaistion nennen, schließlich glaubte er selbst schon an seine Verwandlung, was ihm sonderbar schmeichelte und ihn auf sehr seltsame Art verwirrte. ,So einfach ist es also, sich selbst zu verlieren', dachte er" (Mann 2006: 171). Im Palast der Kandake, der mit orientalisierenden Klischees beschrieben wird, kommt es zur Verführung durch die lüsterne Gastgeberin mit üppigem Essen und Drogen (Zynda 1986: 70). Alexander wehrt sich zuerst, indem er sich auf Hephaistions zurückhaltende Wesensart beruft, doch Kandake hat ihn durchschaut und appelliert an ihn als sinnesfreudigen Alexander: „'Du bist nicht du', sagte sie plötzlich... „Bist du nicht gerne du?’“ Die Methode ist erfolgreich: „Der Kampf des Ichs galt nicht mehr“ (Mann 2006: 174). Ernüchtert verspürt Alexander am nächsten Morgen zwar Gewissensbisse, weil er den Namen seines Freundes missbraucht hat, verbirgt dies aber hinter einer Fassade der Schroffheit.

Im Folgenden baut er eine noch größere Distanz zu Hephaistion auf, der in ihm nur noch das „entstellte Bild des Freundes“ sieht (Mann 2006: 190), und lässt ihn schließlich, als er schwer erkrankt, alleine sterben. Erst angesichts des Toten erkennt er, was er an ihm verloren hat: „Nur er, Alexander, hatte sich verändert; denn Hephaistion sah wie immer aus, nur etwas schöner" (Mann 2006: 206). Die Einsamkeit, die Alexander zuvor immer in Abwehr seines Alter Egos gesucht hatte, ist nun durch dessen Ableben vollständig. Als Alexander wenig später stirbt, wird, wie in einer Reminiszenz zum Schicksal des Dorian Gray, erneut der Unterschied zwischen ihm und Hephaistion deutlich, den der Erfolg nicht negativ gezeichnet hatte. Der Engel des Todes mit christologischem Bezug (Härle 1988: 300), der den sterbenden Herrscher aufsucht, stellt fest: „Alexander, dein junges Gesicht ist verwüstet, was für hässliche Falten. Und die Haut ist ganz schlaff“ (Mann 2006: 224). Es liegt somit eine Darstellung des getrennten, gegeneinander kämpfenden Ichs mit seinem Alter Ego vor.

Ein völlig anderes Bild der Beziehung zwischen Alexander und Hephaistion zeichnet der französische Schriftsteller Roger Peyrefitte. Seine Darstellung ist im Kontext seines Engagements für die Homosexuellenbewegung in Frankreich zu sehen (Berger 2010: 105758; J ackson 2006), die eine artifizielle griechische Antike (Makedonien eingeschlossen) als Ideal, etwa in Anlehnung an die nostalgisch verklärenden Bildwelten der Fotografien von Wilhelm von Gloeden, beschwor (Goldman 2006: 237-38). Peyrefittes Bekenntnis zur Päderastie, ersichtlich auch aus seiner Korrespondenz mit "his friend and fellow pederast" (Golsan 1986: 88) Henry de Montherlant (Peyrefitte/Sipriot 1983: 43), schlägt sich in seinem Alexanderroman - eine überhöhende Verklärung der Knabenliebe - nieder. So beschwören die beiden jungen Makedonen regelmäßig die aristotelische Freundschaftslehre und, als ein Leitmotiv, die Parallele zum homerischen Paar, da die 
„Liebe, die Alexander und Hephaistion füreinander empfanden, Spiegelbild der Liebe zwischen Achilles und Patroklos“ ist (Peyrefitte 1980: I, 254). Diese Heroenimitatio wird in sexualisierter Hinsicht interpretiert: Alexander und Hephaistion verbindet eine erotische Beziehung, seit sie dreizehn sind. Schon zwei J ahre zuvor hatte Alexander ihm öffentlich seine Liebe erklärt (Peyrefitte 1980: I, 36, 105-108).

Die Darstellung Hephaistions als des „anderen Alexanders“ steht unter dem Zeichen ihrer Ebenbürtigkeit: Sie haben eine identische Ausbildung genossen, sind gleich anmutig, begehrenswert, sportlich, fähig im Kampf und beliebt (Peyrefitte 1980: I, 13, 33, 310). Hephaistions Doppelgängerrolle wird noch dadurch betont, dass er am gleichen Tag Geburtstag hat wie Alexander, ähnlich gekleidet ist, die gleichen Geschenke erhält, von Olympias wie ein zweiter Sohn begrüßt wird und sich Alexanders Geliebte Kampaspe mit ihm teilt (Peyrefitte 1980: I, 9, 13, 278-9, 310, 325; II, 652, 750).

Ganz anders als bei Klaus Mann sind ihre Gefühle wechselseitig. Alexander lässt sich Hephaistions Liebe wiederholt versichern, nimmt sie nicht als selbstverständlich hin und fühlt sich von ihr so beflügelt, dass auch seine Taten unter ihrem Zeichen stehen:

Ich besitze die Liebe und begehre den Ruhm, damit er seinen Widerschein auf meine Liebe werfe und sie noch verschöne (...) Ich schenke dir mein Königreich (...) Du gibst mir Kraft, so wie Patroklos Achilles Kraft gab.' - „Mein Königreich, das bist du', erwiderte Hephaistion (Peyrefitte 1980: I, 114; II, 763).

Das unterschwellige Problem des unübersehbaren Bedeutungsunterschieds zwischen Alexander und seinem Alter Ego - trotz Hephaistions Aufstieg blieb er doch „nur“ der Gefolgsmann des Herrschers, der als Eroberer eines Weltreichs in die Geschichte einging - löst Peyrefitte mit einer Aufwertung seiner Hephaistionfigur als Alexanders Retter. So bewahrte Hephaistion ihn einst bei einer Jagd vor dem Tod, erscheint bei ihren gemeinsamen Schiffsreisen als sein potentieller Retter, da Alexander nicht schwimmen kann, und soll für den Fall, dass Alexander sich als zeugungsunfähig erweist, stellvertretend für einen Erben sorgen (Peyrefitte 1980: I, 33, 104, 324).

In Oliver Stones von der Fachwelt viel kritisierten Film Alexander aus dem Jahr 2004 - „an unproductive model of historiophotic semiauthenticity“ (Nisbet 2010: 218) - stellt gerade die Beziehung zwischen Alexander und Hephaistion eines der vielen missglückten Elemente dar (Ogden 2009: 201). Alexander als „an iconic figure and as a hero“ (Baynham 2009: 294), Kriegsheld und Eroberer, unterhält zu seinem Freund und Waffengefährten eine von halbherziger Ambivalenz geprägte Beziehung irgendwo zwischen Kameradschaft und diffuser, undefinierter Anziehung. Wie tief ihre Beziehung möglicherweise sein könnte, wird durch einige tiefe Blicke, „manly hugs“ (Baynham 2009: 300), verhalten eifersüchtiges Mienenspiel angesichts von Nebenbuhlern und das Geschenk eines Rings allenfalls angedeutet, um dann aufkeimende Ahnungen wieder in den Hintergrund zu drängen (Nisbet 2010: 209). Zwar operieren beide mit der Achilles-Patroklos-Parallele, doch scheint dies mehr auf das Rollenmodell der Waffengefährten abzuzielen. Inwieweit eine sexuelle Komponente impliziert war, bleibt unausgesprochen. Signifikant für 
Hephaistions vagen Status als Alexanders „maybe-kind-of-boyfriend” (Nisbet 2010: 230) ist die seltsam verkrampfte Szene an seinem Sterbebett, das Stone von Ekbatana nach Babylon verlegt und Hephaistion mit Typhus kämpfen lässt. Nicht minder konträr zu den antiken Zeugnissen ist, dass Alexander ihn noch lebend antrifft und die wenige, ihm verbleibende Zeit zu einem letzten Gespräch nutzt. Dabei wirkt jedoch äußerst befremdlich, dass er sich von dem Sterbenden abwendet, räumlich auf weite Distanz geht und während ihres Dialogs auch noch konsequent aus dem Fenster schaut, als wäre er mit den Gedanken ganz woanders. Gerade hinsichtlich der Bedeutung des Tods für antike heroische Paare fällt das Versagen von Stones Alexander in diesem entscheidenden Moment besonders ins Gewicht:

Death is the climax of the friendship, the occasion of the most extreme expressions of tenderness (...) and it weds them forever. Indeed, it is not too much to say that death is to friendship what marriage is to romance (Halperin 1990: 79).

Unter dieser Prämisse wirkt Alexanders eigentümlich abwesende Verabschiedung von dem sterbenden Freund eher wie die Scheidung. Dennoch wird in dieser Szene das Doppelgängermotiv mit Hephaistion als Alexanders - gutem - Alter Ego am deutlichsten beschworen:

Alexander: You saved me from myself. Please don't leave me, Hephaistion. (...)

Hephaistion: I worry for you without me.

Alexander: I am nothing without you (...) We will die together (...) It's our destiny (Nisbet 2010: 228).

Gefärbt vom Rollenmodell des Achilles und seines unabwendbaren Tods, wird deutlich, dass der Doppelgänger ohne seinen anderen Teil nicht leben kann. Stirbt das Alter Ego, muss das Ego zwangsläufig folgen. Allerdings erscheint Stones Hephaistion, gespielt von Jared Leto, nicht wie ein eigenständiges Komplement, sondern wie ein Schatten des Herrschers ohne eigene Meinung. Zudem wirkt er mit den sehr langen Haaren, geflochtenen Zöpfen und üppig mit Kajal geschminkten Augen - Effeminierung als bildsprachlicher Code für eine „Orientalisierung" nach Gaugamela - wie ein androgyner oder sogar effeminierter weicherer Gegenpart des von Colin Farrell verkörperten Alexanders, der ihn somit noch viriler dastehen lässt. Diese betonte Maskulinität mag dazu gedient haben, seine für einen Hollywoodhelden unübliche Eskapade mit dem Eunuch Bagoas zu relativieren. Somit ist Hephaistion am ehesten als der schwache Teil Alexanders zu sehen, der letztendlich jedoch immer wieder zu seinem dominierend maskulinen Part zurückfindet.

\section{FAZIT}

Das Motiv des „doppelten Alexanders“ in Gestalt seines Freundes Hephaistion, das wohl eine spätere Ausgestaltung und kein Teil von Alexanders eigener 
Repräsentationspolitik gewesen war, wurde zu einem Standardmotiv in der antiken Alexanderliteratur. Dabei galt die Szene mit der Verwechslung der Freunde durch Sisygambis als exemplum für Großmut, Freundschaft und Milde. Im Zuge der Negativporträtierung Alexanders als Tyrann in Teilen der antiken Tradition wurde auch sein Alter Ego unvorteilhaft dargestellt. Ihre kurz nacheinander erfolgten Tode galten als schicksalhaft miteinander verknüpfte Ereignisse; Hephaistions Ableben wurde als Vorzeichen für Alexanders Ende gedeutet.

In der Kunst der europäischen Renaissance und des Barock wurde dem Motiv ein neues Element hinzugefügt: das gleiche Aussehen des doppelten Alexanders als bildsprachlicher Code. Anhand der exemplarischen Beispiele für Alexanderbiographien des 20. J ahrhunderts lassen sich zwei diametral entgegengesetzte Konzepte des doppelten Alexanders fassen: Hephaistion als opportunistischer, unfähiger und intriganter Schmeichler, dessen Karriere kein gutes Licht auf die Personalpolitik seines Förderers wirft wie bei Schachermeyr, oder Hephaistion als positive Ergänzung Alexanders, der ihn zu seinen Erfolgen beflügelt, wie bei Lane Fox.

In der Populärkultur findet sich die Variante Klaus Manns, der in psychologisierender Deutung Hephaistion als positiven Part zeigt, der sich nicht von dem Erfolg verbiegen lässt, während Alexander in einer Entfremdung von seinem Alter Ego zum Tyrannen wird. Oliver Stone folgt in seinem Film dem positiven Modell, verleiht ihm aber so wenig Profil, dass sein Konzept verschwommen bleibt.

Bei allen behandelten Beispielen aus dem 20. und 21. J ahrhundert ist indes das Motiv der zusammenhängenden Todesfälle der beiden, ebenso wie in den antiken Quellen, virulent. Das Ableben des Alter Egos ist eine Vorschau auf Alexanders Ende. Man kann daher folgern, dass sich das antike Konzept des doppelten Alexanders, vermutlich vor allem durch Kleitarchos vertreten, zumindest in dieser Hinsicht durchgesetzt hat.

\section{BIBLIOGRAPHIE}

Ameling, Walter (1988). "Alexander und Achilleus", in Wolfgang Will und Johannes Heinrichs (Hg.), Zu Alexander d. Gr. FS G. Wirth, II, Amsterdam: Hakkert, 657-92.

Atkinson, John E. (1980). A commentary on Q. Curtius Rufus' Historiae Alexandri Magni, Books 3 and 4. Amsterdam/ Uithoorn: J .C. Gieben.

- (2009). Curtius Rufus. Histories of Alexander the Great, Book 10. Introduction and Historical Commentary. Oxford: OUP.

Badian, Ernst (1976). "Review: Robin Lane Fox, Alexander the Great”, J ournal of Hellenic Studies 96: 229-30.

—. (1998). "Hephaistion”, Der Neue Pauly 5: 350.

Baynham, Elizabeth (1998). Alexander the Great. The unique history of Quintus Curtius. Ann Arbor: The University of Michigan Press. 
—. (2009). "Power, Passion and Patrons. Alexander, Charles LeBrun and Oliver Stone”, in Waldemar Heckel and Lawrence A. Tritle (Hg.), Alexander the Great. A new history. Oxford: Wiley-Blackwell: 294-310.

Berger, Helmut (2010). "Peyrefitte, Roger", Biographisch-Bibliographisches Kirchenlexikon 31: 1057-61.

Berve, Helmut (1926). Das Alexanderreich auf prosopographischer Grundlage II. Prosopographie. München: Beck.

Birkenholz, A. Thomas (2002). Die Alexander-Geschichte von Charles Le Brun. Historische und stilistische Untersuchung der Werkentwicklung. Bern: Peter Lang.

Bosworth, Albert Brian (1980). A historical Commentary on Arrian's History of Alexander, I. Oxford: Clarendon.

Briant, Pierre (2009). Alexander the Great and his Empire. Princeton: PUP.

Brosius, Maria (2003). “Alexander and the Persians", in J oseph Roisman (Hg.), Brill's Companion to Alexander the Great. Leiden: Brill: 169-93.

Carney, Elizabeth D. (1975). Alexander the Great and the Macedonian aristocracy. Clemson: Duke University.

Cocke, Richard (1978). 'Veronese's ,Family of Darius' at the National Gallery”, The Burlington Magazine 120: 325-29.

Goldman, Jason (2006). "The Golden Age of Gay Porn: Nostalgia and the Photography of Wilhelm von Gloeden", GLQ: A J ournal of Gay and Lesbian Studies 12: 237-58.

Golsan, Richard J . (1986). "Review Essay: A Montherland Renaissance", Rocky Mountain Review of Language and Literature 40: 87-91.

Grell, Chantal/ Michel, Christian (1988). L'École des Princes ou Alexandre disgracié. Essai sur la mythologie monarchique de la France absolutiste. Paris: Les Belles Lettres.

Habicht, Christian (1956). Gottmenschentum und griechische Städte. München: Beck.

Hadjinicolaou, Nicos (1997). "The disputes about Alexander and his glorification in the visual arts", in Nicos Hadjinicolaou (Hg.), Alexander the Great in European art. Thessaloniki: Organisation for the Cultural Capital of Europe: 15-24.

Härle, Gerhard (1988). Männerweiblichkeit. Homosexualität bei Thomas und Klaus Mann. Frankfurt a.M.: Athenäum.

Halperin, David M. (1990). "Heroes and their pals”, in David M. Halperin, One hundred years of Homosexuality. New York/ London: Routledge: 75-87.

Hammond, Nicholas G.L. (1993). Sources for Alexander the Great. An analysis of Plutarch's Lives and Arrian's Anabasis Alexandrou. Cambridge: CUP.

Heckel, Waldemar (1978). "The somatophylakes of Alexander the Great. Some thoughts", Historia 27: 224-28.

—. (1985). "The boyhood friends of Alexander the Great”, Emerita 53: 285-89.

—. (1992). The marshals of Alexander's empire. London and New York: Routledge. 
—. (2009). Who's who in the empire of Alexander the Great. Oxford: OUP.

—. (2011). "Alexander, Achilles, and Heracles", in: Elizabeth Baynham (Hg.), Essays in honour of A.B. Bosworth (i.D.).

Heißerer, Dieter (2006). "Nachwort", in: Mann, Klaus (2006). Alexander. Roman der Utopie. 2. Auflage. Reinbek: Rowohlt: 229-48.

Hölscher, Tonio (2009). Herrschaft und Lebensalter: Alexander der Große: Politisches Image und anthropologisches Modell. Basel: Schwabe.

Jackson, Julian (2006): "Sex, Politics and Morality in France, 1954-1982", History Workshop J ournal 61: 77-102.

J affé, Michael (1963). “Le Brun at Versailles”, The Burlington Magazine 105: 558-59.

J ones, Christopher P. (1986). Culture and society in Lucian. Cambridge, Mass./London: Harvard University Press.

J ones, Roger/ Penny, Nicholas (1983). Raphael. London: Yale University Press.

Konstan, David (1997). Friendship in the classical world. Cambridge: CUP.

Kirchner, Thomas (2001). Der epische Held. Historienmalerei und Kunstpolitik im Frankreich des 17. J ahrhunderts. München: Fink.

Kraft, Konrad (1965). "Der behelmte Alexander der Große“, J ahrbuch für Numismatik und Geldgeschichte 15: 6-32.

Krause, Katharina (1997). “Die Kamele Eliezers und die Elephanten des Porus. Typologie und ,Parallèle' in Historien von Nicolas Poussin, Sébastien Bourdon und Charles Le Brun“, Marburger J ahrbuch für Kunstwissenschaft 24: 213-30.

Lane Fox, Robin (1974). Alexander der Große. Eine Biographie. Übers. V. Peter Zentner u. Peter Dering. Düsseldorf: Claasen.

LeRider, Georges (2003). Alexandre le Grand. Monnaie, finances et politique. Paris: PUF. Mann, Klaus (2006). Alexander. Roman der Utopie. 2. Auflage. Reinbek: Rowohlt.

McKechnie, Paul (1995): “Diodorus Siculus and Hephaistion’s Pyre“, Classical Quarterly 45: 418-32.

Meuus, Alexander (2009). „Some institutional problems concerning the succession of Alexander the Great", Historia 58: 287-310.

Müller, Sabine (2003). Maßnahmen der Herrschaftssicherung gegenüber der makedonischen Opposition bei Alexander dem Großen. Frankfurt am Main: Peter Lang.

—. "Rezension: Robin Lane Fox, Alexander der Große", Sehepunkte 5, Nr. 4 <http:/ / www.sehepunkte.historicum.net/ 2005/04/ 7745.html>.

- (2006). "Alexander der Große als neuer Achilles", in Stefan Jaeger und Christer Petersen (Hg.), Zeichen des Krieges in Literatur, Film und den Medien II. Kiel: Steven Ludwig Verlag: 263-94. 
—. (2011). “Q. Curtius Rufus”, in: Bagnall, Roger u.a. (Hg.), Encyclopedia of Ancient History. Oxford: Wiley-Blackwell (i.D.).

Naumann, Uwe (Hg.) (2001): „Ruhe gibt es nicht, bis zum Schluß“. Klaus Mann (19091946). Bilder und Dokumente. Reinbek: Rowohlt.

Nisbet, Gideon (2010). "And your father sees you”. Paternity in Alexander (2004), in Elizabeth D. Carney and Daniel Ogden (Hg.), Philip II and Alexander the Great. Father and son, lives and afterlives. Oxford: OUP: 217-31.

Noll, Thomas (2005). Alexander der Große in der nachantiken bildenden Kunst. Mainz: von Zabern.

Ogden, Daniel (2009). “Alexander's sex life”, in Waldemar Heckel and Lawrence A. Tritle (Hg.), Alexander the Great. A new history. Oxford: Wiley-Blackwell: 203-17.

Palagia, Olga (2000). "Hephaestion's pyre and the royal hunt of Alexander", in Albert Brian Bosworth und Elizabeth Baynham (Hg.), Alexander the Great in fact and fiction. Oxford: OUP: 167-206.

Peyrefitte, Roger (1980). Der junge Alexander. I-II. Übers. v. Sybille A. Rott-Illfeld, München: Goldmann.

—. et Sipriot, Pierre (1983). Henry Montherlant/Roger Peyrefitte. Correspondence. Paris: Robert Laffant.

Pöschl, Sabine (1988). “Alexander Magnus Maximus - neue Aspekte zur Ikonographie Alexanders des Großen im Quattrocento“, Römisches J ahrbuch für Kunstgeschichte 23-24: 62-74.

Posner, Donald (1959). "Charles Lebrun's Triumphs of Alexander", The Art Bulletin 41: 237-48.

Reames-Zimmerman, J eanne (1998). Hephaistion Amyntoros: Eminence grise at the court of Alexander the Great. Pennsylvania: Pennsylvania State University.

- (1999). "An atypical affair? Alexander the Great, Hephaistion Amyntoros and the nature of their relationship", Ancient History Bulletin 13: 81-96.

Schachermeyr, Fritz (1973). Alexander der Große. Das Problem seiner Persönlichkeit und seines Wirkens. Wien: Verlag der Österreichischen Akademie der Wissenschaft.

Schmitter, Amy M. (2002). "Representation and the body of power in French Academic painting", J ournal of the History of Ideas 63: 399-424.

Schwarzenberg, Erkinger (1969). "From the Alessandro Morente to the Alexandre Richelieu. The portraiture of Alexander the Great in seventeenth-century Italy and France", J ournal of the Warburg and Courtauld Institutes 32: 398-405.

Stadter, Philip A. (1980). Arrian of Nicomedia. Chapel Hill: University of North Carolina Press.

Starn, Randolph (1986). "Reinventing heroes in Renaissance Italy", Journal of Interdisciplinary History 17: 67-84. 
Stewart, Andrew (1993). Faces of power. Alexander's image and Hellenistic politics. Berkeley: Berkeley University Press.

-. (2003). "Alexander the Great in Greek and Roman Art", in J oseph Roisman (Hg.), Brill's Companion to Alexander the Great, Leiden: Brill 2003: 31-66.

Stoneman, Richard (2008). Alexander the Great. A Life in Legend. New Haven und London: Yale University Press.

Tarn, William Woodthorpe (1968). Alexander der Große. Darmstadt: WBG.

Terribile, Claudia (2009). Del piacere della virtue. Paolo Veronese, Alessandro Magno e il patriziato veneziano. Marsilio: Venice.

Vickers, Michael (1999). "The changing image of Alexander the Great", in Martin Henig und Dimitris Plantzos (Hg.), Classicism to Neo-classicism. Essays dedicated to Gertrud Seidmann. Oxford: OUP: 29-37.

Wilson-Chevalier, Kathleen (1997). "Alexander the Great of Fontainebleau", in: Hadjinicolaou, Nicos (Hg.), Alexander the Great in European art. Thessaloniki: Organisation for the Cultural Capital of Europe: 25-33.

Wirth, Gerhard (1967). "Hephaistion", in Der Kleine Pauly 2: 1022-23.

—. (1985). "Besprechung: F. Schachermeyr, Alexander der Große", in: Gerhard Wirth, Studien zur Alexandergeschichte. Darmstadt: WBG: 280-88.

—. (1993). Der Brand von Persepolis. Folgerungen zur Geschichte Alexanders des Großen. Amsterdam: Hakkert.

Zynda, Stefan (1986). Sexualität bei Klaus Mann. Bonn: Bouvier. 